Retrospective Study

\title{
e Comparison of Percutaneous Transforaminal Endoscopic Discectomy and Microendoscopic Discectomy for the Surgical Management of Symptomatic Lumbar Disc Herniation: A Multicenter Retrospective Cohort Study with a Minimum of 2 Years' Follow-Up
}

Panfeng Yu, MD, PhD , Pengfei Zan, MD, PhD³, Xifeng Zhang, MD², Hua Qiang, MD, Rebekah M. Samsonraj, $\mathrm{PhD}^{4}$, Jiaguang Tang, MD, PhD ${ }^{1}$, and Peng Huang, MD, $\mathrm{PhD}^{2}$

\footnotetext{
From: ${ }^{2}$ Department of Orthopedics, Beijing Tongren Hospital, Capital Medical University, Beijing, China; 2Department of Orthopedics, Chinese PLA General Hospital, Beijing, China; ${ }^{3}$ Department of Orthopedics, Shanghai General

Hospital affiliated to Jiaotong

University, Shanghai, China;

${ }^{4}$ Department of Orthopedics, Mayo Clinic, Rochester, MN, USA

Address Correspondence: Peng Huang, MD, PhD Department of Orthopedics Chinese PLA General Hospital No. 28 Fuxing Road Haidian District Beijing, China

E-mail: zjuooı@hotmail.com

Disclaimer: Panfeng Yu and Pengfei Zan contributed equally to this work. There was no external funding in the preparation of this manuscript.

Conflict of interest: Each author certifies that he or she, or a member of his or her immediate family, has no commercial association (i.e., consultancies, stock ownership, equity interest, patent/licensing arrangements, etc.) that might pose a conflict of interest in connection with the submitted manuscript.

Manuscript received: o1-01-2019

Revised manuscript received: 05-14-2019

Accepted for publication: 05-21-2020

Free full manuscript: www.painphysicianjournal.com

Background: Percutaneous transforaminal endoscopic discectomy (PTED) and microendoscopic discectomy (MED) are alternative minimally invasive procedures for the treatment of symptomatic lumbar disc herniation (LDH). However, insufficient literature exists to highlight the differences between the procedures.

Objectives: This study intended to clarify whether PTED results in better clinical outcomes compared with MED in the surgical management of single-level LDH.

Study Design: A multicenter retrospective cohort study.

Setting: This study took place in 2 spinal minimally invasive centers in Beijing, China.

Methods: A multicenter retrospective study was conducted in consecutive patients diagnosed with symptomatic LDH receiving PTED or MED in 2 spinal minimally invasive centers from April 2009 to July 2016. A total of 1,053 patients were recruited, of which 632 underwent PTED and 421 underwent MED. All patients were followed with a minimum of 2 years; a set of clinical outcomes were extracted and analyzed.

Results: The operation time was similar between groups $(71.2 \pm 15.1$ minutes in the PTED group and $69.4 \pm 12.5$ minutes in the MED group; $P=0.518$ ); length of incision was significantly shorter; intraoperative blood loss was less in the PTED group $(P<0.001)$; hospital stay was $3.6 \pm 1.5$ days in the PTED group and $5.4 \pm 2.8$ days in the MED group with significant differences detected $(P$ $=0.018)$; however, intraoperative fluoroscopy was longer with significantly higher cost with the PTED group $(P<0.001)$. Transient dysesthesia and wound complications were more common in the MED group ( $P=0.039$ and $P=0.026$, respectively); however, no significant differences were found with total complications $(P=0.139)$. Significant lower Visual Analog Scale pain score (back and leg) were detected on day 1 postoperatively $(P=0.007$ and $P=0.018$, respectively). No significant differences were found at all other time points $(P>0.05)$. Significantly better Oswestry Disability Index (ODI) score was detected postoperatively at 1 month in the PTED group (19.6 \pm 9.8 vs. 27.2 $\pm 9.3 ; P=0.016) ; \mathrm{ODI}$ score at other time points did not differ significantly between groups $(P>$ 0.05). Modified MacNab criteria showed that most patients experienced excellent and good results with no significant differences between groups $(P=0.511)$.

Limitation: This was a multicenter retrospective study wherein the surgeons may have introduced bias to the study.

Conclusions: Both PTED and MED present to be an acceptable long-term efficacy for the treatment of LDH. Although PTED is associated with longer intraoperative fluoroscopy and a little more cost, it should still be considered superior to MED considering the benefits of lesser invasion, shorter hospital stays, quicker pain relief, and functional recovery.

Key words: Percutaneous transforaminal endoscopic discectomy, microendoscopic discectomy, lumbar disc herniation, VAS score, ODI score

Pain Physician 2021: 24:E117-E125
} 
umbar disc herniation (LDH), one of the most common orthopedic issues, is associated with a morbidity of $20 \%$ to $35 \%$ typically in populations aged older than 50 years $(1,2)$. Symptoms range from lower back pain, lumbosacral radiculopathy of different degrees, and limb activity disorders. Approximately $40 \%$ to $60 \%$ of these patients merit a surgical intervention when conservative management has failed $(3,4)$. Surgical procedures utilized to treat symptomatic $\mathrm{LDH}$ include traditional open discectomy (OD), microdiscectomy (MD), and more recently the minimally invasive techniques including percutaneous transforaminal endoscopic discectomy (PTED) and microendoscopic discectomy (MED) (5).

The surgical procedure of OD for the treatment of LDH was first introduced in 1934 (6) and performed with a standard surgical incision, which generally involved a laminar or hemilaminar discectomy.With the introduction of the microscope, the traditional OD was improved to an open MD under a smaller incision (7). More minimally invasive techniques, such as PTED and MED, are current prevailing procedures widely performed owing to the advantages of considerably lesser trauma and faster rehabilitation (8). MED was first described by Perez-Cruet and Forely in 1997 (9) and was performed through a transmuscular approach using tubular retractors under the visualization of a microendoscope.Numerous studies have demonstrated its safety and efficacy even in treating recurrent $\operatorname{LDH}(10,11)$. PTED, introduced by Yeung and Tsou (12) in 2002, which combines the endoscope and radiofrequency techniques organically, has been claimed with the potential benefits of minimal bleeding, easier anesthesia, reduced complication rates, and faster postoperative recovery $(13,14)$.

Considering that PTED and MED are both regarded as safe and effective techniques with a similar indication in treating symptomatic $\operatorname{LDH}(4,5,15)$, surgeons always encounter a dilemma in choosing an optimal procedure in between. To date, the relative benefits and risks of PTED and MED have not been well established. Thus we conducted this multicenter retrospective clinical study with a large cohort recruitment to clarify whether PTED would yield preferable clinical outcomes in comparison to MED in the treatment of symptomatic LDH.

\section{Methods}

\section{Patient Selection}

This multicenter retrospective clinical case-control study was reviewed and approved by the ethics committee of Beijing Tongren Hospital affiliated with Capital Medical University and the Chinese PLA General Hospital. All procedures involving human patients were performed in accordance with the Declaration of Helsinki. From April 2009 to July 2016, all consecutive patients who were diagnosed with symptomatic LDH and treated by PTED or MED in the 2 spinal minimally invasive centers were retrospectively reviewed. Surgeries were performed by 2 experienced spinal surgeons at each center. Surgical techniques were selected by patients after thorough education on the pros and cons of the 2 minimally invasive procedures.

Inclusion criteria were as follows: (1) patients with symptomatic back pain and/or radiating leg pain; (2) symptoms lasting for more than 6 weeks, in which conservative treatment was ineffective; (3) computed tomography and magnetic resonance imaging indicating a single-level $\mathrm{LDH}$, which correlated with the clinical symptoms; and (4) a minimum of 2 years' follow-up. Exclusion criteria included: (1) LDH combining spinal canal stenosis, spondylolisthesis, or segmental instability; (2) recurrent LDH; (3) coexisting pathological conditions such as tumor, active infection, immunosuppression, severe obesity (body mass index $\geq 35.0 \mathrm{~kg} / \mathrm{m}^{2}$ ), or lumbar fracture, or (4) history of spinal surgery.

\section{Data Collection}

First, demographic information of all patients was accessed. Relevant perioperative data, including operation time, intraoperative blood loss, length of incision, intraoperative fluoroscopy, total cost, and complications were reviewed and recorded in detail. Clinical measurements were collected starting from preoperative time point until 2 years' follow-up. Pain (back and leg) was evaluated by using the Visual Analog Scale (VAS) score at day 1 postoperatively and later followup visits at 1, 3, and 6 months, and at 1- and 2-year outpatient visiting; daily life was assessed by the Oswestry Disability Index (ODI) separately at a follow-up of 1, 3, and 6 months, and at 1- and 2-year outpatient visiting. The modified MacNab criteria, classified as excellent, good, fair, and poor, was utilized to evaluate patients' satisfaction at 2 years postoperatively.

\section{Surgical Techniques}

The surgeons in each center were formally trained and highly experienced to perform PTED and MED procedures. The surgeons strictly adhered to the standard surgical procedures. 


\section{PTED Group}

In the PTED group, surgeries were performed under regional anesthesia in the prone position with the Transforaminal Endoscopic Spine System (TESSYS). The skin entrance point was located superior to the iliac crest approximately 10 to $14 \mathrm{~cm}$ from the midline. After administration of local infiltration of $0.5 \%$ lidocaine, the 18-gauge puncture needle was introduced to the lateral foramen under the guidance of x-ray examination. A 22-gauge needle was then inserted into the center of the intervertebral disc, followed by the injection of contrast medium (iohexol with methylene blue at the ratio of 3:1). A guidewire was inserted through the 18 -gauge needle, and a $0.8-\mathrm{cm}$ incision was made at the margin of the guidewire. Cannulated dilators were consecutively inserted, and reamers were used to dilate the bony foramen. Finally, the PTED was connected through the working channel and continuous irrigation with $0.9 \% \mathrm{NaCl}$ solution to keep the view clear. Then the blue-stained degenerated nucleus pulposus was identified and removed by endoscopic forceps until nerve root was adequately decompressed. Surgical sheaths were removed after meticulous homeostasis, and finally suturing was performed.

\section{MED Group}

In the MED group, the procedures were performed under general anesthesia in prone positioning. After confirming the surgical level by fluoroscopy, an approximately $1.8-\mathrm{cm}$ incision was made $1.5 \mathrm{~cm}$ from the midline, a K-wire was introduced to the junction of the inferior part of the lamina and medial facet joint. Multiple levels of dilators were consecutively inserted, and an operative tubular retractor was fixed. The microendoscope was then attached to the tubular retractor. Soft tissue and partial ligamentum flavum was resected to expose the dural sac and nerve root. The extruded disc was removed with disc forceps until the nerve root was sufficiently decompressed. Tubular retractor was finally removed, and a drainage tube was placed after meticulous hemosta- sis. Working routeway was irrigated and tissue sutured back in layers. Drainage tube was normally removed the second morning.

\section{Statistical Analyses}

All statistical analyses were performed using SPSS 19.0 (IBM Corporation, Armonk, NY). Categorical variables were presented as numbers with frequencies. The $\chi^{2}$ test was used to detect the differences between groups. Continuous data were presented as mean with standard deviation, Student t-test or the MannWhitney test was used to compare the differences. The analysis of variance (ANOVA) was also performed for data involving multiple factors, such as VAS score and ODI during the follow-up time points. A $P$ value $<0.05$ was considered statistically significant.

\section{Results}

A total of 1,208 patients were reviewed and given full consideration during the research period. A total of 112 patients were initially excluded based on the inclusion and exclusion criteria, and during data collection course, 43 cases were excluded because important data were missing. A total of 1,053 patients were identified in the final study, among which 632 patients receiving PTED procedure constituted the PTED group and 421 patients receiving MED treatment were named the MED group. A flow diagram is depicted in Fig. 1. Standard examples of pre- and postoperative magnetic resonance images of the patients receiving PTED and MED are shown as Figs. 2 and 3.

The patients' demographic information and

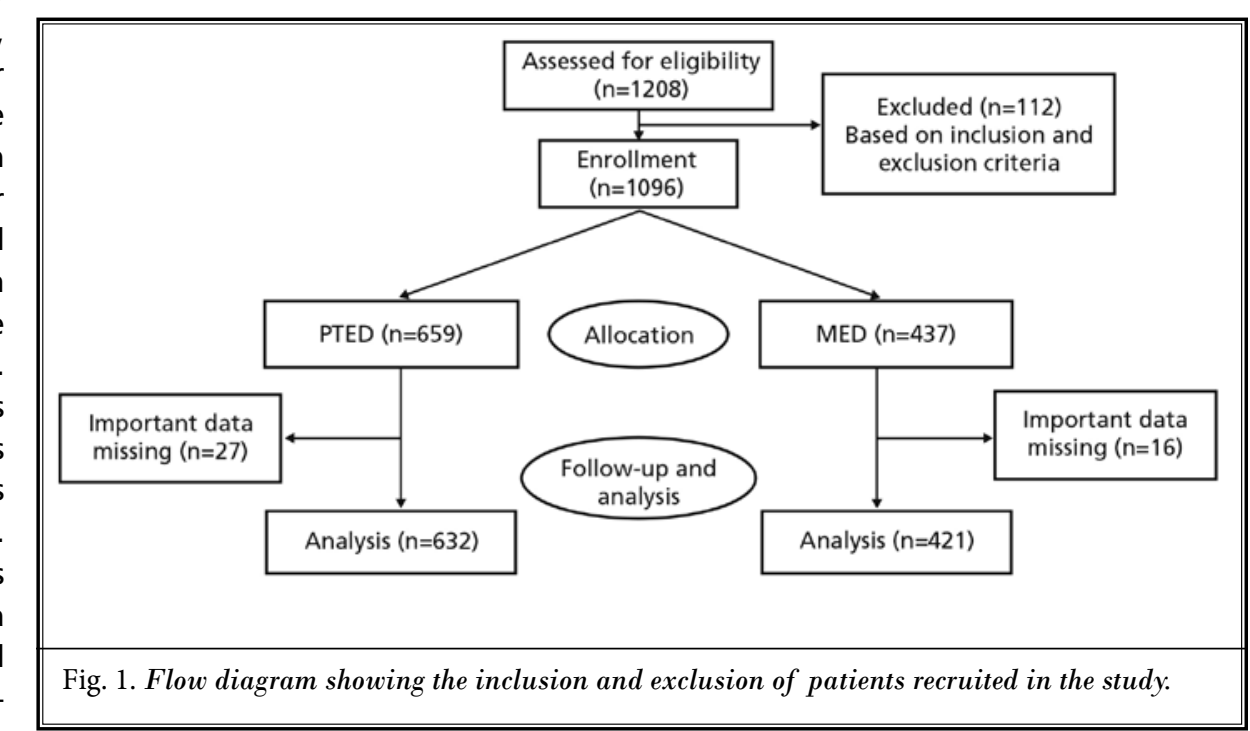


preoperative characteristics are presented in Table 1 with no significant differences detected between the groups. The mean age of the cohort was 40.8 years, and female patients accounted for $44.6 \%$ of the total. The most common type of disc herniation was paramedian and lateral, and L4/5, L5/S1 were the most commonly affected surgical levels. The mean follow-up period was $32.3 \pm 6.8$ months in the PTED group and $31.7 \pm 7.2$ months in the MED group.
Clinical outcome measurements are presented in Table 2. The operation time was $71.2 \pm 15.1$ minutes in the PTED group and $69.4 \pm 12.5$ minutes in the MED group, with no significant difference detected $(P=$ 0.518 ). Length of incision was significantly shorter in the PTED group $(P<0.001)$; and intraoperative blood loss was $18.6 \pm 6.3 \mathrm{~mL}$ in the PTED group, which was significantly less than $45.2 \pm 21.8$ in the MED group $(P<$ $0.001)$. Significant difference was detected in duration
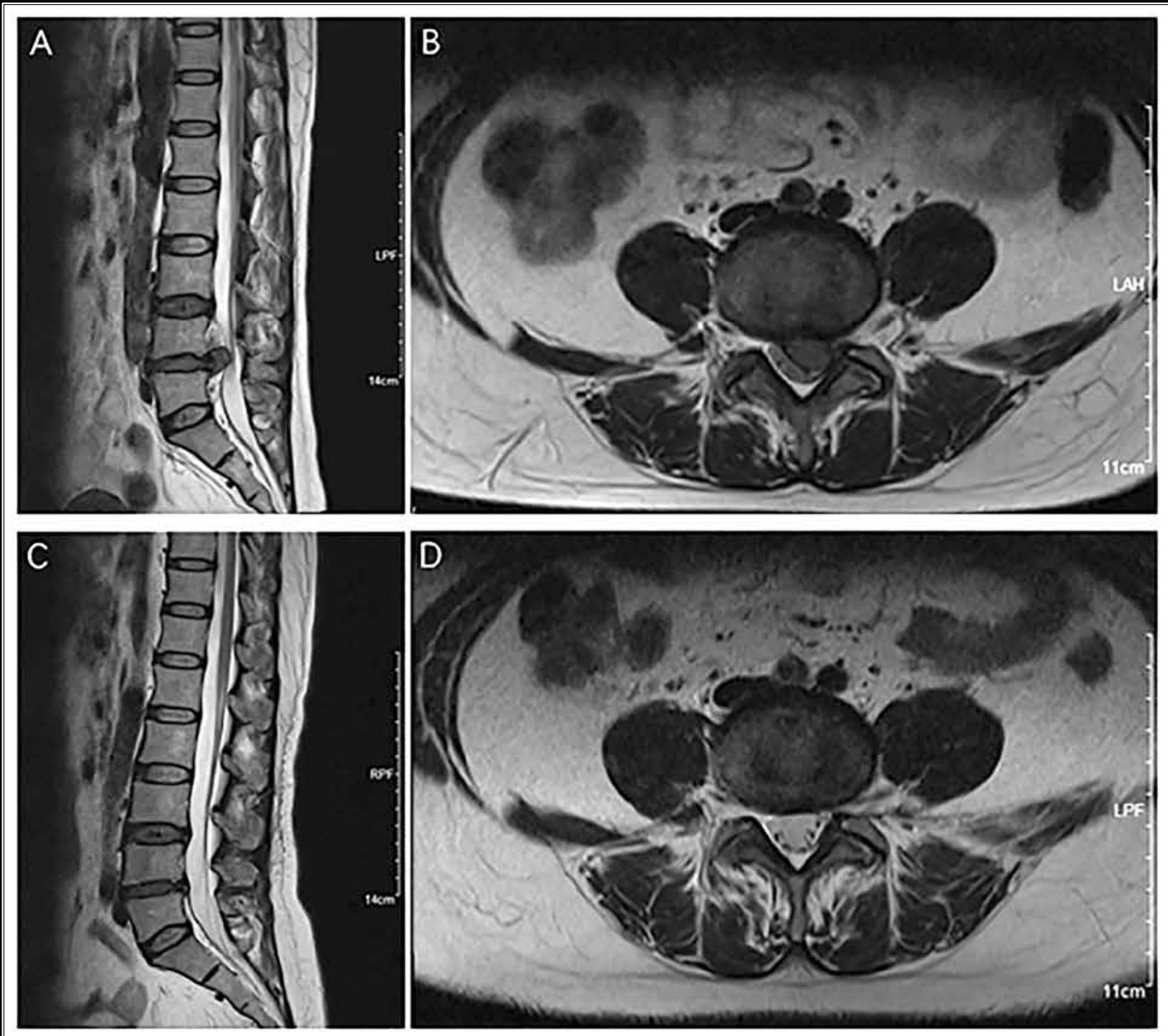

Fig. 2. A 39-year-old female patient with severe left sciatica and lower back pain was treated with PTED. (A and B) Preoperative magnetic resonance images showing severe L4/L5 disc herniation; ( $C$ and D) magnetic resonance images obtained 2 years after surgery showing that the protruded disc was removed with completed decompression.

L: left; A: anterior; P: posterior; F: foot; H: head; LAH: left anterior head; LPF: left posterior foot 


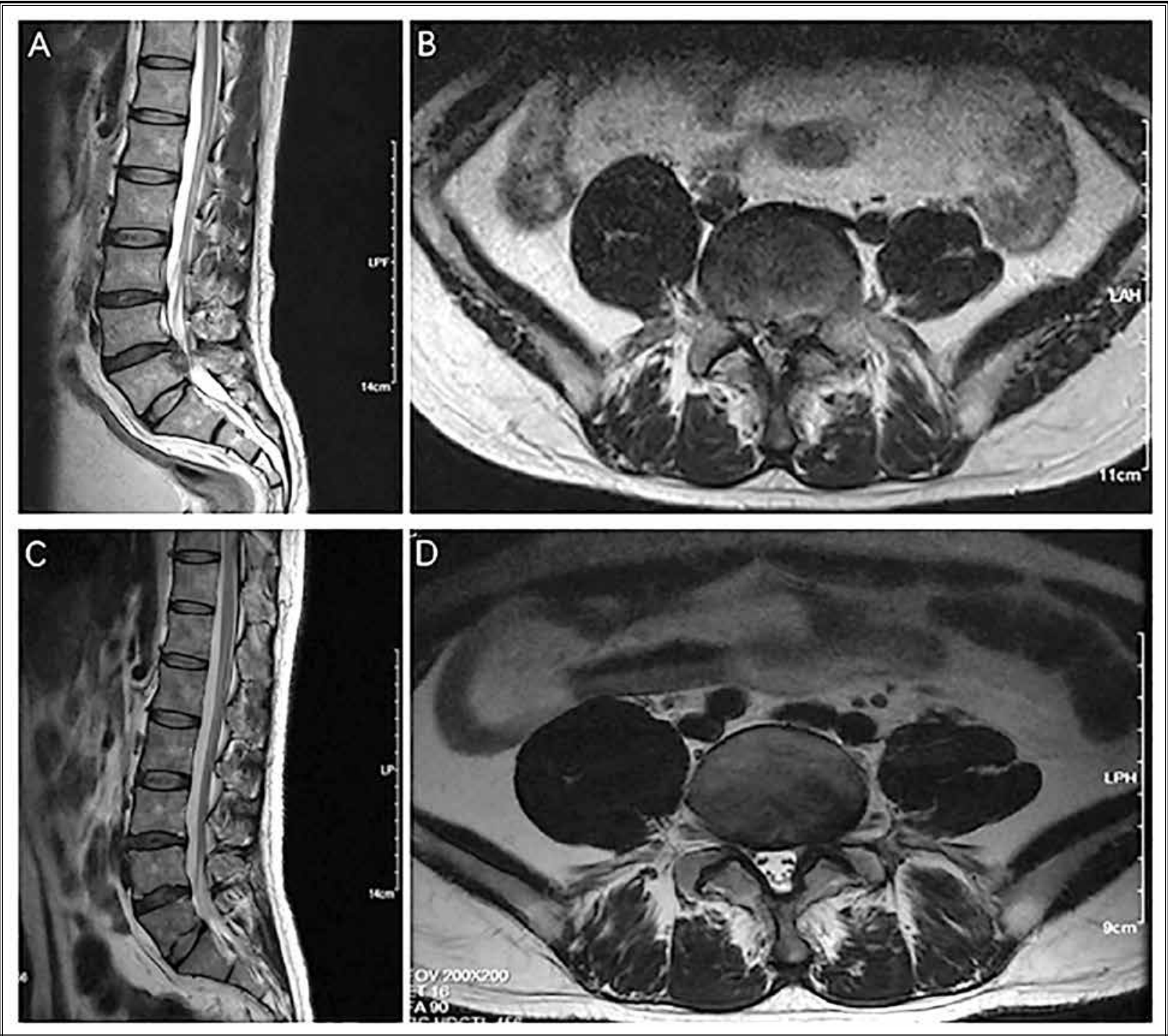

Fig. 3. A 36-year-old male patient who experienced lower back pain and severe left sciatica with leg numbness for 6 months was treated with MED. (A and B) Preoperative magnetic resonance images showing severe L4/L5 disc herniation; ( $C$ and D) magnetic resonance images obtained 2 years after surgery showing that the protruded disc was removed with completed decompression.

L: left; A: anterior; P: posterior; F: foot; H: head; LAH: left anterior head; LPF: left posterior foot

of hospital stay with $3.6 \pm 1.5$ days in the PTED group and $5.4 \pm 2.8$ days in the MED group $(P=0.018)$. However, intraoperative fluoroscopy was $12.7 \pm 3.6$ seconds in the PTED group, which was significantly longer than $4.4 \pm 1.3$ seconds in the MED group $(P<0.001)$. Furthermore, total cost was $\$ 3.888 .4 \pm \$ 686.9$ USD in the PTED group and $\$ 2,203.3 \pm \$ 537.4$ USD in the MED group, which was statistically significant $(P<0.001)$.

A total of $63(10.0 \%)$ cases encountered complica- tions in the PTED group and $54(12.8 \%)$ cases in the MED group, with no significant differences detected $(P$ $=0.139$ ). Rate of residue and recurrence was similar between groups ( $4.3 \%$ vs. $5.0 \%$, respectively in the PTED and MED groups, $P=0.585)$, transient dysesthesia was more common in the MED group $(3.3 \%$ vs. $1.4 \%, P=$ 0.039 ), and more wound complications were shown in the MED group than in the PTED group ( 8 vs. 3 cases, $P=$ 0.026). No significant differences were found in the rate 
of nerve root injury and dural tear. Rate of revision was $3.5 \%$ in the PTED group and $4.0 \%$ in the MED group, with no significant differences between the groups $(P=0.639)$ (Table 3).

Preoperative VAS score including back pain and leg pain did not differ between the groups. Significant differences were detected on the VAS back pain score on day 1 postoperatively $(2.1 \pm 1.6$ scores in the PTED group,

Table 1. Baseline demographic information of patients (PTED vs. MED).

\begin{tabular}{|l|c|c|c|}
\hline Item & $\begin{array}{c}\text { PTED } \\
(\mathbf{n = 6 3 2})\end{array}$ & $\begin{array}{c}\text { MED } \\
(\mathbf{n = 4 2 1})\end{array}$ & $\begin{array}{c}\text { P } \\
\text { value }\end{array}$ \\
\hline Age (yrs) & $41.2 \pm 11.8$ & $40.1 \pm 12.4$ & 0.736 \\
\hline Gender (male/female) & $345 / 287$ & $238 / 183$ & 0.534 \\
\hline BMI (kg/m $\left.{ }^{2}\right)$ & $24.5 \pm 3.7$ & $24.2 \pm 3.3$ & 0.448 \\
\hline Disease duration (months) & $4.8 \pm 3.2$ & $5.0 \pm 3.4$ & 0.661 \\
\hline Type of disc herniation & $56(8.9 \%)$ & $39(9.3 \%)$ & 0.823 \\
\hline Median & $308(48.7 \%)$ & $198(47.0 \%)$ & 0.588 \\
\hline Paramedian & $237(37.5 \%)$ & $161(38.2 \%)$ & 0.808 \\
\hline Lateral & $31(4.9 \%)$ & $23(5.5 \%)$ & 0.688 \\
\hline Far lateral & $66(10.4 \%)$ & $48(11.4 \%)$ & 0.624 \\
\hline Surgical segment & $352(55.7 \%)$ & $226(53.7 \%)$ & 0.520 \\
\hline L3/4 or higher & $214(33.9 \%)$ & $147(34.9 \%)$ & 0.723 \\
\hline L4/5 & $5.2 \pm 1.8$ & $5.4 \pm 2.1$ & 0.774 \\
\hline L5/S1 & $6.3 \pm 2.2$ & $6.0 \pm 1.9$ & 0.841 \\
\hline VAS score & $66.8 \pm 15.9$ & $31.7 \pm 7.2$ & 0.811 \\
\hline Back & \multicolumn{5}{|l}{} \\
\hline Leg & \multicolumn{5}{|l}{} \\
\hline ODI score & \multicolumn{5}{|l}{} \\
\hline \hline
\end{tabular}

BMI, body mass index.

Table 2. Clinical outcome measurements (PTED vs. MED).

\begin{tabular}{|l|c|c|c|}
\hline Parameter & $\begin{array}{c}\text { PTED } \\
(\mathbf{n}=\mathbf{6 3 2})\end{array}$ & $\begin{array}{c}\text { MED } \\
(\mathbf{n}=\mathbf{4 2 1})\end{array}$ & P value \\
\hline $\begin{array}{l}\text { Operation time } \\
\text { (minutes) }\end{array}$ & $71.2 \pm 15.1$ & $69.4 \pm 12.5$ & 0.518 \\
\hline Length of incision (cm) & $0.8 \pm 0.1$ & $1.7 \pm 0.4$ & $<0.001^{*}$ \\
\hline $\begin{array}{l}\text { Intraoperative blood } \\
\text { loss (mL) }\end{array}$ & $18.6 \pm 6.3$ & $45.2 \pm 21.8$ & $<0.001^{*}$ \\
\hline $\begin{array}{l}\text { Intraoperative } \\
\text { fluoroscopy (seconds) }\end{array}$ & $12.7 \pm 3.6$ & $4.4 \pm 1.3$ & $<0.001^{*}$ \\
\hline Hospital stay (days) & $3.6 \pm 1.5$ & $5.4 \pm 2.8$ & $0.018^{*}$ \\
\hline Total cost (USD\$) & $3888.4 \pm 686.9$ & $2203.3 \pm$ & 537.4 \\
\hline
\end{tabular}

${ }^{\star}$ Indicates significant difference. and $3.5 \pm 1.9$ scores in the MED group; $P=0.007)$ and VAS leg pain score $(1.9 \pm 2.1$ scores in the PTED group, and $3.1 \pm 2.2$ scores in the MED group; $P=0.018$ ); no significant differences were found at other time points. Further ANOVA analysis failed to detect significant differences on the VAS back and leg pain score with time included as a variable $(P=0.156$ and $P=0.525$, respectively). Detailed data are provided in Table 4 and Fig. 4.

ODI scores are shown in Table 5, and the trends with follow-up time are presented in Fig. 5. Significantly better ODI scores were detected at 1 month postoperatively in the PTED group $(19.6 \pm 9.8$ vs. $27.2 \pm 9.3, P=$ 0.016 ) with no significant differences detected at other follow-up time points. Further ANOVA analysis also failed to demonstrate a significant difference between the groups $(P=0.278)$. Based on the MacNab criteria, most patients experienced excellent and good results, with a rate of $92.6 \%$ in the PTED group and $91.4 \%$ in the MED group, showing no significant differences $(P=$ 0.511 ; Table 6).

\section{Discussion}

Currently, PTED and MED are alternative procedures for the surgical treatment of symptomatic LDH with no definitive conclusions regarding their differences. The findings of the present study indicate that both PTED and MED are effective and safe, which can be acceptable in the management of LDH. Compared with MED, PTED is associated with shorter incision and shorter hospital stay, lesser intraoperative blood loss, better early pain relief and functional recovery Although combining with longer intraoperative fluoroscopy and a little more cost, PTED should still be considered superior to MED for the treatment of LDH.

MED is carried out based on the combination of traditional posterior interlaminar fenestration surgery and the endoscopic technique, making it possible to achieve

Table 3. Postoperative complications (PTED vs. MED).

\begin{tabular}{|l|c|c|c||}
\hline Parameter & $\begin{array}{c}\text { PTED } \\
(\mathbf{n = 6 3 2})\end{array}$ & $\begin{array}{c}\text { MED } \\
(\mathbf{n = 4 2 1})\end{array}$ & $\begin{array}{c}\boldsymbol{P} \\
\text { Value }\end{array}$ \\
\hline Residue or recurrence & $27(4.3 \%)$ & $21(5.0 \%)$ & 0.585 \\
\hline Transient dysesthesia & $9(1.4 \%)$ & $14(3.3 \%)$ & $0.039^{\star}$ \\
\hline Nerve root injury & $11(1.7 \%)$ & $5(1.2 \%)$ & 0.472 \\
\hline Dural tear & $13(2.1 \%)$ & $6(1.4 \%)$ & 0.451 \\
\hline Wound complications & $3(0.5 \%)$ & $8(1.9 \%)$ & $0.026^{\star}$ \\
\hline Total & $63(10.0 \%)$ & $54(12.8 \%)$ & 0.139 \\
\hline Revision cases & $22(3.5 \%)$ & $17(4.0 \%)$ & 0.639 \\
\hline
\end{tabular}

${ }^{\star}$ Indicates significant difference. 
Comparison of PTED and MED for Lumbar Disc Herniation

Table 4. VAS pain scores (back and leg) (PTED vs. MED).

\begin{tabular}{|l|c|c|c|c|c|c|c||}
\hline \hline $\begin{array}{l}\text { Groups/Time } \\
\text { Point }\end{array}$ & Preoperative & I Day PO & 1 Month PO & 3 Months PO & 6 Months PO & 1 Year PO & 2 Years PO \\
\hline PTED (back) & $5.2 \pm 1.8$ & $2.1 \pm 1.6$ & $1.7 \pm 1.3$ & $1.3 \pm 1.1$ & $0.7 \pm 1.0$ & $0.5 \pm 1.2$ & $0.4 \pm 1.0$ \\
\hline MED (back) & $5.4 \pm 2.1$ & $3.5 \pm 1.9$ & $1.9 \pm 1.2$ & $1.6 \pm 1.0$ & $0.9 \pm 1.1$ & $0.7 \pm 0.9$ & $0.6 \pm 0.8$ \\
\hline$P$ value & 0.774 & $0.007^{*}$ & 0.352 & 0.515 & 0.368 & 0.416 & 0.223 \\
\hline PTED (leg) & $6.3 \pm 2.2$ & $1.9 \pm 2.1$ & $1.2 \pm 1.6$ & $1.1 \pm 1.3$ & $0.7 \pm 1.2$ & $0.5 \pm 1.1$ & $0.5 \pm 1.2$ \\
\hline MED (leg) & $6.0 \pm 1.9$ & $3.1 \pm 2.2$ & $1.3 \pm 1.5$ & $1.2 \pm 1.4$ & $0.7 \pm 1.1$ & $0.4 \pm 1.3$ & $0.5 \pm 1.1$ \\
\hline$P$ value & 0.841 & $0.018^{*}$ & 0.882 & 0.761 & 0.877 & 0.784 & 0.863 \\
\hline
\end{tabular}

$\mathrm{PO}$, postoperative; ${ }^{\star}$ indicates significant difference.

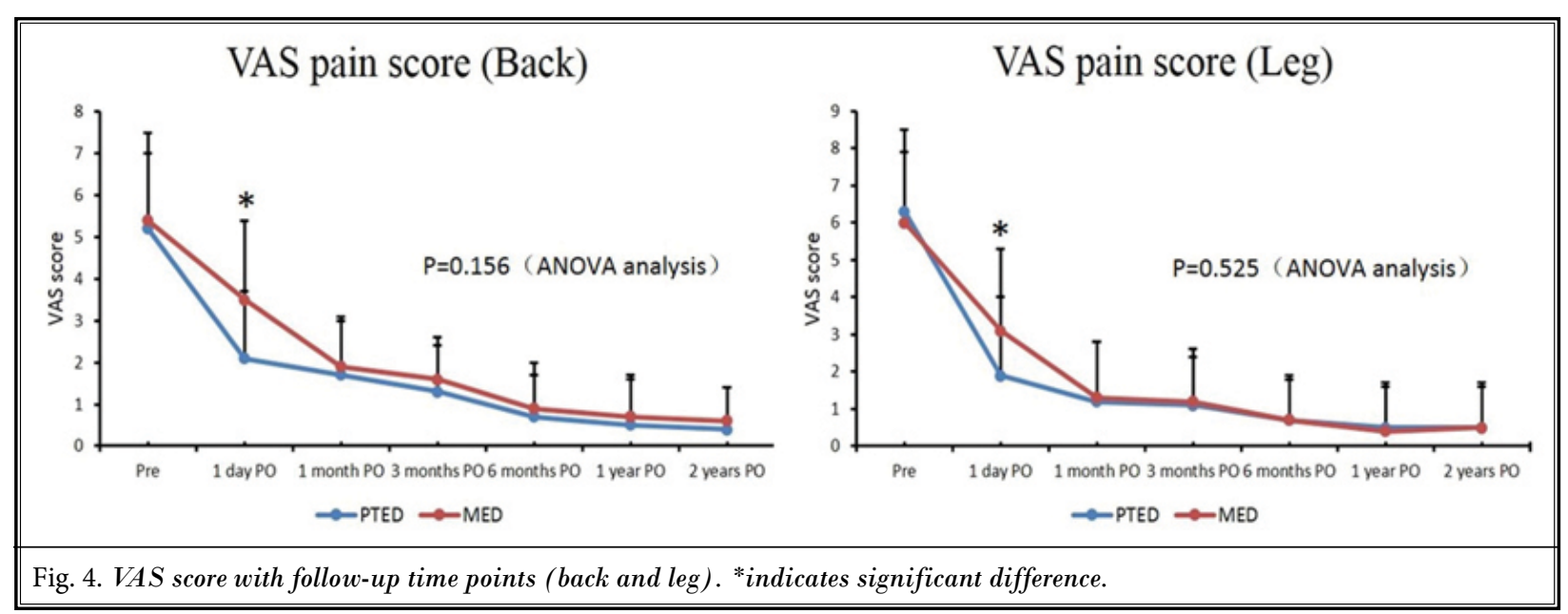

Table 5. ODI score (PTED vs. MED).

\begin{tabular}{|l|c|c|c|}
\hline Time Point & $\begin{array}{c}\text { PTED } \\
(\mathbf{n = 6 3 2})\end{array}$ & $\begin{array}{c}\text { MED } \\
(\mathbf{n = 4 2 1})\end{array}$ & P Value \\
\hline Preoperative & $66.8 \pm 15.9$ & $68.1 \pm 16.3$ & 0.619 \\
\hline 1 month PO & $19.6 \pm 9.8$ & $27.2 \pm 9.3$ & $0.016^{*}$ \\
\hline 3 months PO & $12.2 \pm 7.6$ & $16.5 \pm 8.1$ & 0.133 \\
\hline 6 months PO & $8.3 \pm 7.7$ & $9.1 \pm 7.4$ & 0.529 \\
\hline 1 year PO & $4.3 \pm 5.5$ & $4.6 \pm 6.1$ & 0.651 \\
\hline 2 years PO & $4.1 \pm 5.9$ & $4.3 \pm 6.4$ & 0.746 \\
\hline
\end{tabular}

PO, postoperative; ${ }^{*}$ indicates significant difference.

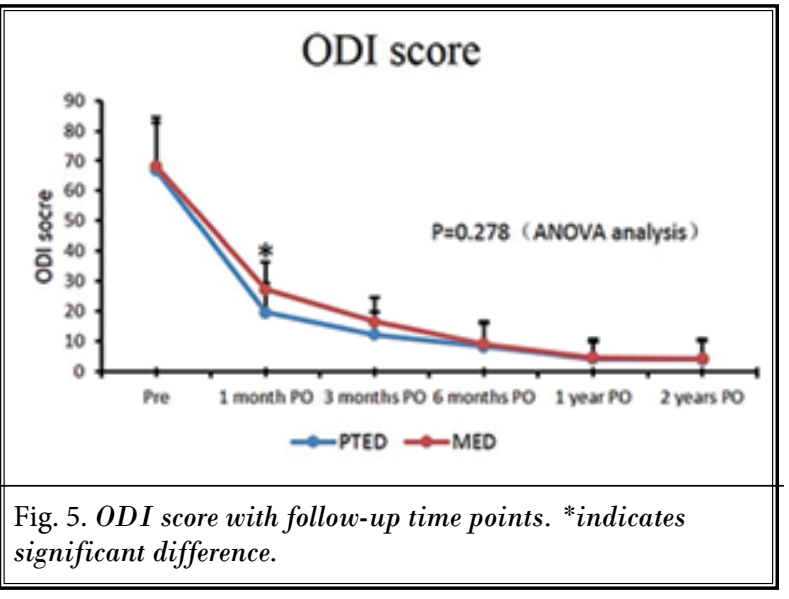

Table 6. Modified MacNab Criteria (PTED vs. MED).

\begin{tabular}{|l|c|c|c|c|c|c||}
\hline Groups & Cases & Excellent & Good & Fair & Poor & Rate (excellent and good) \\
\hline PTED & 632 & $312(49.4 \%)$ & $273(43.2 \%)$ & $33(5.2 \%)$ & $14(2.2 \%)$ & $92.6 \%$ \\
\hline MED & 421 & $218(51.8 \%)$ & $167(39.7 \%)$ & $24(5.7 \%)$ & $12(2.9 \%)$ & $91.4 \%$ \\
\hline$\chi^{2}$ value & & 0.589 & 1.293 & 0.113 & 0.423 & 0.432 \\
\hline$P$ value & & 0.443 & 0.255 & 0.736 & 0.515 & 0.511 \\
\hline
\end{tabular}


adequate decompression by a shorter incision $(10,11)$. With the assistance of the microendoscope, the surgical field is enlarged, and the risk of nerve and blood vessel injury is decreased during the decompression (16). Moreover, MED involves limited damage of the stabilizing structures, such as the paraspinal muscles and ligamentum flavum, which may potentially provide benefits over traditional open surgery (17). In a case series of 873 patients, MED proved to be an effective microendoscopic system with fine long-term outcome in treating LDH (18). PTED approach combines the endoscope and radiofrequency techniques organically and achieves direct extraction of the diseased disc by a 7.5-mm working tunnel. As one of the newest minimally invasive techniques, PTED has become more popular among the spinal surgeons and achieved satisfactory outcomes $(13,14,19)$. In the current study, owing to the natural differences between the techniques, the incision length is shorter, intraoperative blood loss is lesser, and cost is higher with the PTED technique, whereas the surgery duration is similar. As PTED can be accomplished under regional anesthesia, postoperative recovery is faster as shown in the differences with hospital stay. However, PTED involves a sightless puncture with a whole course of intraoperative fluoroscopy, resulting in longer radiation exposure.

The reported recurrence rate of PTED was approximately $8 \%$, which was comparable with that of $5 \%$ in MED (20), and both recurrence rates are also comparable with conventional fenestration surgery (21). In the current study, the rate of residue or recurrence was similar to the previous data, which did not show a significant difference between groups. Total complications and revision cases were also similar, thus indicating that although PTED was regarded as a more minimally invasive procedure, it did not show advantages over MED with respect to complications. However, it is important to note that the transient dysesthesia is more common in the MED group probably owing to the traversing of the nerve root and retraction of the dural sac; and the rate of wound complications is higher as a larger incision is required in the MED technique. It has been reported that the risks of nerve root injury and dural tear in the PTED are $2 \%$ and $1.1 \%$, respectively, which are similar to those of $\operatorname{MED}(22,23)$, the current study corresponds to the published data.

MED is performed via a posterior approach with multifidus split by dilators (24), whereas PTED is highly minimally invasive with a less than $1-\mathrm{cm}$ incision with posterior structures preserved $(13,25)$. Lower back pain and lumbosacral radicular pain are the typical characteristics of LDH. The current study demonstrated that both PTED and MED can be acceptable considering postoperative pain relief. Compared with MED, PTED experienced better early pain relief both on the back and leg; long-term pain relief was similar between the 2 techniques. ODI, a daily life assessing tool, is widely used to evaluate patients with spinal disorders, and the correlation of ODI score with pain scores has been demonstrated by previous study (26). The current study showed that ODI score was significantly improved during the follow-up time points. ODI score was significantly better at 1-month postoperative evaluation in the PTED group, which may be contributed by the better early pain relief. The modified MacNab criteria is also useful for the assessment of patients with LDH. The current study indicated that the excellent and good results obtained at 2 years' follow-up time point were similar between techniques, thereby correlating with previously published data (27).

Although a well-designed retrospective study was conducted, several limitations are to be noted. One major limitation is the innate flaw existing with retrospective studies in which information may be not accurately recorded. Another possible limitation includes the factor of this work being a multicenter study, wherein the surgeons at each center may have introduced bias to the results. Third, the fact that surgical technique was chosen by patients may have effects on the postoperative outcome measurements. Despite these limitations, the current study recruited the largest cohort to date, which demonstrates that PTED technique might be a preferable treatment modality for the management of symptomatic LDH.

\section{Conclusions}

Both PTED and MED present to be an acceptable long-term efficacy for the treatment of symptomatic LDH. Although PTED is associated with longer intraoperative fluoroscopy and an associated slightly higher cost, it should still be considered superior to MED considering the advantages of lesser invasion, shorter hospital stays, quicker pain relief and functional recovery. Further prospective randomized controlled trials may provide additional validations to the findings reported in this study. 


\section{RefERENCES}

1. Shin JS, Lee J, Kim MR, et al. The shortterm effect of integrated complementary and alternative medicine treatment in inpatients diagnosed with lumbar intervertebral disc herniation: A prospective observational study. J Altern Complement Med 2016; 22:533-543.

2. Song HP, Sheng HF, Xu WX. A casecontrol study on the treatment of protrusion of lumbar intervertebral disc through PELD and MED. Exp Ther Med 2017; 14:3708-3712.

3. Patel DR, Kinsella E. Evaluation and management of lower back pain in young athletes. Transl Pediatr 2017; 6:225-235.

4. Bruggeman AJ, Decker RC. Surgical treatment and outcomes of lumbar radiculopathy. Phys Med Rehabil Clin N Am 2011; 22:161-177.

5. Amin RM, Andrade NS, Neuman BJ. Lumbar disc herniation. Curr Rev Musculoskelet Med 2017; 10:507-516.

6. Gruber P, T Böni. Sciatica: From stretch rack to microdiscectomy. Unfallchirurg 2015;118(suppl 1):53-65.

7. Sørlie A, Gulati S, Giannadakis C, et al. Open discectomy vs microdiscectomy for lumbar disc herniation--A protocol fora pragmatic comparative effectiveness study. F100oRes 2016; 5:2170.

8. Telfeian AE, Iprenburg M, Wagner R. Endoscopic spine surgery: Distance patients will travel for minimally invasive spine surgery. Pain Physician 2017; 20:E145-E149.

9. Perez-Cruet MJ, Foley KT, Isaacs RE, et al. Microendoscopic lumbar discectomy: Technical note. Neurosurgery 2002; 51(5 suppl):S129-S136.

10. Hubbe U, Franco-Jimenez P, Klingler JH, Vasilikos I, Scholz C, Kogias E. Minimally invasive tubular microdiscectomy for recurrent lumbar disc herniation. J Neurosurg Spine 2016; 24:48-53.
11. He J, Xiao S, Wu Z, Yuan Z. Microendoscopic discectomy versus open discectomy for lumbar disc herniation: A meta-analysis. Eur Spine J 2016; 25:1373-1381.

12. Yeung AT, Tsou PM. Posterolateral endoscopic excision for lumbar disc herniation: Surgical technique, outcome, and complications in 307 consecutive cases. Spine 2002; 27:2081-2082.

13. Gadjradj PS, Harhangi BS. Percutaneous transforaminal endoscopic discectomy for lumbar disk herniation. Clin Spine Surg 2016; 29:368-371.

14. Ning $H X$, Yuan YW, Zhang QY, Sun ZZ, Ning HY, Wang P. Percutaneous transforaminal endoscopic discectomy and miniincision surgery in the treatment of lumbar intervertebral disc protrusion. J Biol Regul Homeost Agents 2018; 32:565-569.

15. Choi G, Lee SH, Bhanot A, Raiturker PP, Chae YS. Percutaneous endoscopic discectomy for extraforaminal lumbar disc herniations: Extraforaminal targeted fragmentectomy technique using working channel endoscope. Spine 2007; 32:93-99.

16. Abudurexiti T, Qi L, Muheremu A, Amudong A. Micro-endoscopic discectomy versus percutaneous endoscopic surgery for lumbar disk herniation. J Int Med Res 2018; 46:3910-3917.

17. Yoon SM, Ahn SS, Kim KH, Kim YD, Cho $\mathrm{JH}$, Kim DH. Comparative study of the outcomes of percutaneous endoscopic lumbar discectomy and microscopic lumbar discectomy using the tubular retractor system based on the VAS, ODI, and SF-36. Korean J Spine 2012; 9:215-222.

18. Wu X, Zhuang $\mathrm{S}$, Mao Z, Chen $\mathrm{H}$. Microendoscopic discectomy for lumbar disc herniation: Surgical technique and outcome in 873 consecutive cases. Spine 2006; 31:2689-2694.
19. Tacconi L, Baldo S, Merci G, Serra G. Transforaminal percutaneous endoscopic lumbar discectomy: Outcome and complications in 270 cases. J Neurosurg Sci 2018 Mar 26. DOI: 10.23736/So390-5616.18.04395-3.

20. $\mathrm{Ng} \mathrm{CY}$, Gibson JN. An aid to the explanation of surgical risks and complications: The International Spinal Surgery Information Sheet. Spine 2011; 36:2333-2345.

21. Kim $\mathrm{CH}$, Chung $\mathrm{CK}$, Park CS, Choi B, Kim MJ, Park BJ. Reoperation rate after surgery for lumbar herniated intervertebral disc disease: Nationwide cohort study. Spine 2013; 38:581-590.

22. Gotfryd A, Avanzi O. A systematic review of randomised clinical trials using posterior discectomy to treat lumbar disc herniations. Int Orthop 2009; 33:11-17.

23. Yong $\mathrm{A}$, Lee $\mathrm{SH}$, Lee JH. Dural tears in percutaneous endoscopic lumbar discectomy. Eur Spine ] 2011; 20:58-64.

24. Patil A, Chugh A, Gotecha $S$, et al. Microendoscopic discectomy for lumbar disc herniations. J Craniovertebr Junction Spine 2018; 9:156-162.

25. Depauw PRAM, Gadjradj PS, Soria van Hoeve JS, Harhangi BS. How do it: Percutaneous transforaminal endoscopic discectomy for lumbar disk herniation. Acta Neurochir (Wien) 2018; 160:2473-2477.

26. Liu $H$, Tao $H$, Luo Z. Validation of the simplified Chinese version of the Oswestry Disability Index. Spine 2009; 34:1211-1216.

27. Sinkemani A, Hong $X$, Gao ZX, et al. Outcomes of microendoscopic discectomy and percutaneous transforaminal endoscopic discectomy for the treatment of lumbar disc herniation: A comparative retrospective study. Asian Spine J 2015; 9:833-840. 
